\title{
A STRENGTHENING OF LETH AND MALITZ'S UNIQUENESS CONDITION FOR SEQUENCES
}

\author{
M. A. KHAMSI AND J. E. NYMANN \\ (Communicated by Andrew M. Bruckner)
}

\begin{abstract}
A series $\sum a_{n}$ of nonnegative real numbers is determined up to a constant multiple by the comparisons of its subsums, provided that $a_{i} \leq$ $\sum_{i>n} a_{i}$ and $\left\{a_{n}\right\}$ decreases to 0 . This characterization is an improvement of Leth and Malitz's results.
\end{abstract}

\section{INTRODUCTION}

Any sequence $\left\{a_{n}\right\}$ of nonnegative numbers induces a preordering on subsets of $N$ in the following way

$$
I \preceq J \quad \text { if and only if } \quad \sum_{i \in I} a_{i} \preceq \sum_{j \in J} a_{j} .
$$

Leth [4] gave conditions on the sequence $\left\{a_{n}\right\}$ under which the induced preordering determines the sequence up to a constant multiple. He proved

Theorem 1. Let $\left\{a_{n}\right\}$ and $\left\{b_{n}\right\}$ be two nonincreasing sequences of real numbers such that

(i) $a_{n}>0, b_{n}>0, \lim _{n \rightarrow \infty} a_{n}=0$, and $\lim _{n \rightarrow \infty} b_{n}=0$,

(ii) $a_{n} \leq r_{n}=\sum_{i>n} a_{i}$ and $b_{n} \leq R_{n}=\sum_{k>n} b_{k}$,

(iii) $\sum_{i \in I} a_{i} \leq \sum_{j \in J} a_{j}$ if and only if $\sum_{i \in I} b_{i} \leq \sum_{j \in J} b_{j}$ for any $I, J \subset N$. Then there is a constant $\alpha$ such that $b_{n}=\alpha a_{n}$ for all $n \in N$.

Note that the condition $a_{n} \leq r_{n}$ in (ii) is satisfied if and only if the set of subsums $E=\left\{\sum \varepsilon_{n} a_{n} ; \varepsilon_{n}=0,1\right\}$ is an interval. For more on the structure of the set $E$ one can consult [3].

This theorem can be seen as a result on purely atomic measures. In fact Chuaqui and Malitz [2] originated this problem by looking for necessary and sufficient conditions for the existence of $\sigma$-additive probability measures compatible with given preorderings.

Malitz [5] has strengthened Leth's result by proving the same conclusion under the weaker assumption

(iii) ${ }^{\prime} \sum_{i \in I} a_{i}=\sum_{j \in J} a_{j}$ if and only if $\sum_{i \in I} b_{i}=\sum_{j \in J} b_{j}$ for all $I, J \subset N$.

Received by the editors February 11, 1991 and, in revised form, August 28, 1991.

1991 Mathematics Subject Classification. Primary 60A05, 40B05, 40A10; Secondary 60B99. 
Recently Nymann [6] extended Leth and Malitz's results when the series $\sum a_{n}$ and $\sum b_{n}$ are convergent.

The main result of this work is to prove the conclusion of Theorem 1 under the weakest assumption

(iii)" $\sum_{I} a_{i}=\sum_{J} a_{j}$ implies $\sum_{I} b_{i}=\sum_{J} b_{j}$ for all $I, J \subset N$.

\section{Preliminaries AND MAIN Result}

The following result can be found in $[4,6]$.

Proposition 1. Let $\left\{a_{n}\right\}$ be a sequence of real numbers. Assume that $0 \leq a_{n+1} \leq$ $a_{n} \leq r_{n}=\sum_{i>n} a_{i}$ for all $n \in N$ and $\lim _{n \rightarrow \infty} a_{n}=0$. Then

(1) For every $0 \leq r \leq \sum_{i=1}^{\infty} a_{i}$, there exists $K \subset N$ such that $r=\sum_{i \in K} a_{i}$. If $r<\sum_{i=1}^{\infty} a_{i}$ then one can assume that $N-K$ is infinite. If $0<r$ then one can assume $K$ is infinite.

(2) There exists $J_{n} \subset(n, \infty)$ such that $a_{n}=\sum_{i \in J_{n}} a_{i}$, with $\min J_{n}=n+1$ for all $n \in N$.

(3) Assume that the series $\sum_{n} a_{n}$ is divergent. Then if $\sum_{I} a_{i}<\sum_{J} a_{j}$, there exists $K \subset N-I$ such that $\sum_{I} a_{i}+\sum_{K} a_{k}=\sum_{J} a_{j}$.

Let us remark that under the assumptions of Proposition 1 if $a_{n}=0$ for some $n$, then $a_{n}=0$ for every $n$. Therefore we will always assume that $a_{n}>0$ for every $n$.

Definition 1. Let $\left\{a_{n}\right\}$ and $\left\{b_{n}\right\}$ be two sequences of nonnegative real numbers. We will write $\left\{b_{n}\right\} \ll\left\{a_{n}\right\}$ if and only if $\sum_{I} a_{i}=\sum_{J} a_{j}$ implies $\sum_{I} b_{i}=\sum_{J} b_{j}$ for all $I, J \subset N$.

The proof of the following lemma can be found in [6].

Lemma 2. Let $\left\{a_{n}\right\}$ and $\left\{b_{n}\right\}$ be two sequences of nonnegative real numbers. Assume that $a_{n+1} \leq a_{n} \leq r_{n}=\sum_{i>n} a_{i}$ for all $n \in N$. Then $b_{n+1} \leq b_{n} \leq$ $\sum_{i>n} b_{i}$ for all $n$, provided that $\left\{b_{n}\right\} \ll\left\{a_{n}\right\}$. Moreover if $\lim _{n \rightarrow \infty} a_{n}=0$ then $\lim _{n \rightarrow \infty} b_{n}=0$.

The proof of the last statement is not given in [6], but it is not hard to deduce it. Indeed, since $\left\{b_{n}\right\}$ is decreasing, $\lim _{n \rightarrow \infty} b_{n}$ exists. Using Proposition 1, one can get $b_{1}=\sum_{J_{1}} b_{j}$ where $J_{1}$ is an infinite subset of $N$. Therefore a subsequence of $\left\{b_{n}\right\}$ converges to 0 , which implies that $\lim b_{n}=0$.

Let us remark that if $\left\{b_{n}\right\} \ll\left\{a_{n}\right\}$ then it is not difficult to see that if $\sum a_{n}$ is divergent then $\sum b_{n}$ is also divergent. In the next proposition we prove the converse.

Proposition 2. Let $\left\{a_{n}\right\}$ and $\left\{b_{n}\right\}$ be two sequences of nonnegative real numbers. Assume that $a_{n+1} \leq a_{n} \leq r_{n}=\sum_{i>n} a_{i}$ and $\sum a_{n}$ is convergent. Then if $\left\{b_{n}\right\} \ll\left\{a_{n}\right\}, \sum b_{n}$ is convergent.

Proof. Assume to the contrary that $\sum b_{n}$ is divergent and $\left\{b_{n}\right\} \ll\left\{a_{n}\right\}$. Define $f$ and $\bar{f}$ by

$$
f\left(\sum_{i \in I} a_{i}\right)=\sum_{i \in I} b_{i}
$$


and

$$
\bar{f}\left(\sum_{i \in I} b_{i}\right)=\sup \left\{\sum_{j \in J} a_{j} ; \sum_{j \in J} b_{j}=\sum_{i \in I} b_{i}\right\} .
$$

Our assumptions on $\left\{a_{n}\right\}$ and $\left\{b_{n}\right\}$ imply that $f$ is defined on $\left[0, \sum a_{i}\right]$ (with values in $[0, \infty])$ and $\bar{f}$ is defined on $[0, \infty]$ (with values in $\left[0, \sum a_{n}\right]$ ). First let us prove that

$$
f(\bar{f}(x))=x \text { for every } x \in[0, \infty) .
$$

Indeed, let $x \in(0, \infty)$. Then by Proposition 1 , the assumptions on $\left\{a_{n}\right\}$ imply that $\bar{f}(x)=\sum_{i \in M} a_{i}$ where $M$ is a subset of $N$. Also if $x=\sum_{I} b_{i}$ then $\bar{f}(x)=\sum_{M} a_{i} \geq \sum_{I} a_{i}$. So we can assume that $M$ is infinite. Set $\delta_{n}=\sum_{M_{n}} a_{i}$ where $M_{n}=M \cap(n, \infty)$ for all $n \in N$. Then by definition of $\bar{f}$ one can find $L \subset N$ such that

$$
\sum_{i \in M} a_{i}-\delta_{n} \leq \sum_{i \in L} a_{i} \leq \sum_{i \in M} a_{i} \quad \text { and } \quad \sum_{i \in L} b_{i}=x .
$$

Clearly we have $\sum_{L} a_{i}-\sum_{\{i \in M ; i \leq n\}} a_{i} \leq \sum_{i>n} a_{i}$. Then by Proposition 1, we obtain

$$
\sum_{i \in L} a_{i}-\sum_{\{i \in M ; i \leq n\}} a_{i}=\sum_{i \in J_{n}} a_{i}
$$

for some $J_{n} \subset N-\{0,1, \ldots, n\}$. Since $\left\{b_{n}\right\} \ll\left\{a_{n}\right\}$, we have

$$
\sum_{i \in L} b_{i}=\sum_{\{i \in M ; i \leq n\}} b_{i}+\sum_{i \in J_{n}} b_{i}=x \text {. }
$$

Then $\sum_{\{i \in M ; i \leq n\}} b_{i} \leq x$ for all $n \in N$. So $\sum_{i \in M} b_{i} \leq x$ holds.

Assume that $\sum_{i \in M} b_{i}<x$. Then the third conclusion of Proposition 1 implies that there exists $K$, a nonempty subset of $N-M$, such that $\sum_{M} b_{i}+$ $\sum_{K} b_{i}=x$. So by the definition of $\bar{f}$ we have

$$
\bar{f}(x) \geq \sum_{i \in M} a_{i}+\sum_{i \in K} a_{i}
$$

which contradicts the fact that $\bar{f}(x)=\sum_{M} a_{i}$. Therefore,

$$
f(\bar{f}(x))=x \text { for all } x \in[0, \infty) .
$$

Next we prove that $\bar{f}$ is strictly increasing. Indeed, let $x_{1}, x_{2} \in[0, \infty)$ with $x_{1}<x_{2}$. Proposition 1(1) implies that $\bar{f}\left(x_{1}\right) \neq \bar{f}\left(x_{2}\right)$. Put $\bar{f}\left(x_{1}\right)=\sum_{M_{1}} a_{i}$ and $\bar{f}\left(x_{2}\right)=\sum_{M_{2}} a_{i}$. Then by using Proposition 1, one can find $K$, a nonempty subset of $N-M_{2}$, such that

$$
\sum_{i \in M_{1}} b_{i}+\sum_{i \in K} b_{i}=\sum_{i \in M_{2}} b_{i}
$$

since $x_{1}=f\left(\bar{f}\left(x_{1}\right)\right)=\sum_{M_{1}} b_{i}$ and $x_{2}=f\left(\bar{f}\left(x_{2}\right)\right)=\sum_{M_{2}} b_{i}$. By the definition of $\bar{f}$ we get

$$
\sum_{i \in M_{1}} a_{i}+\sum_{i \in K} a_{i} \leq \bar{f}\left(x_{2}\right)=\sum_{i \in M_{2}} a_{i}
$$

which implies that $\bar{f}\left(x_{1}\right)=\sum_{M_{1}} a_{i}<\bar{f}\left(x_{2}\right)$. 
The last step consists of proving that $\bar{f}$ is continuous. In order to show that $\bar{f}$ is left-continuous (resp. right-continuous) at $x \in(0, \infty)$, it is enough to prove that for some $x_{n}<x$ (resp. $x_{n}>x$ ) with $\lim _{n \rightarrow \infty} x_{n}=x$ then $\lim _{n \rightarrow \infty} \bar{f}\left(x_{n}\right)=\bar{f}(x)$ because $\bar{f}$ is increasing.

Left-continuity is easy to show. Indeed, let $x \in(0, \infty)$. Then $\bar{f}(x)=\sum_{M} a_{i}$ where $M$ is an infinite subset of $N$. Since $f(\bar{f}(x))=x$, we have $x=\sum_{M} b_{i}$. Set $\delta_{n}=\sum_{i \in M_{n}} b_{i}$ and $x_{n}=x-\delta_{n}$ where $M_{n}=\{i \in M ; i>n\}$ for all $n \in N$. Then clearly we have $\lim _{n \rightarrow \infty} x_{n}=x$ and $\lim \bar{f}\left(x_{n}\right)=\bar{f}(x)$, since

$$
\sum_{\{i \in M ; i \leq n\}} a_{i} \leq \bar{f}\left(x_{n}\right)<\bar{f}(x) .
$$

So $\bar{f}$ is left-continuous on $(0, \infty)$.

To complete the proof of continuity of $\bar{f}$, let us show that $\bar{f}$ is rightcontinuous.

Let $x \in(0, \infty)$, and again set $\bar{f}(x)=\sum_{M} a_{i}$. Since $x=\sum_{M} b_{i}$, one can assume that $N-M$ is infinite. Put $N-M=\left\{k_{1}, k_{2}, \ldots\right\}$ and $x_{n}=x+b_{k_{n}}$ for all $n \in N$. Then $\lim _{n \rightarrow \infty} x_{n}=x$. Since $\left\{x_{n}\right\}$ is decreasing, $\left\{\bar{f}\left(x_{n}\right)\right\}$ is a decreasing sequence with $\bar{f}\left(x_{n}\right) \geq \bar{f}(x)$ for all $n$. Then $\lim _{n \rightarrow \infty} \bar{f}\left(x_{n}\right)=\omega$ exists and $\omega \geq \bar{f}(x)$. Our assumptions on $\left\{a_{n}\right\}$ imply that $\omega=\sum_{I} a_{i}$ for some subset $I \subset N$. Since $\omega>0$, one can assume that $N-I$ is infinite. Set

$$
\omega_{n_{0}}=\omega+\sum_{\left\{i \in N-I ; i>n_{0}\right\}} a_{i}
$$

for $n_{0} \in N$. Then one can find $l_{0} \in N$ such that $\bar{f}\left(x_{n}\right) \leq \omega_{n_{0}}$ for all $n \geq l_{0}$. Since $\omega \leq \bar{f}\left(x_{n}\right)$ for all $n$, we get that

$$
\bar{f}\left(x_{n}\right)=\sum_{\left\{i \in I ; i \leq n_{0}\right\}} a_{i}+\sum_{i \in J_{n}} a_{i}
$$

for some $J_{n} \subset N-\left\{0,1,2, \ldots, n_{0}\right\}$ for all $n \geq l_{0}$. Then

$$
x_{n}=f\left(\bar{f}\left(x_{n}\right)\right)=\sum_{\{i \in I ; i \leq n\}} b_{i}+\sum_{i \in J_{n}} b_{i}
$$

for all $n \leq l_{0}$. In particular, $\sum_{\left\{i \in I ; i \leq n_{0}\right\}} b_{i} \leq x_{n}$ holds for all $n \geq l_{0}$, which implies

$$
x=\lim x_{n} \geq \sum_{\left\{i \in I ; i \leq n_{0}\right\}} b_{i} .
$$

Since this is true for all $n_{0} \in N$, we get $\sum_{I} b_{i} \leq x$. And because

$$
\omega=\sum_{i \in I} a_{i} \leq \bar{f}\left(\sum_{i \in I} b_{i}\right) \leq \bar{f}(x) \leq \sum_{i \in I} a_{i}=\omega,
$$

we get $\bar{f}(x)=\omega=\lim \bar{f}\left(x_{n}\right)$. So the proof of the continuity of $\bar{f}$ is complete.

Therefore $\bar{f}((0, \infty))$ is an interval. It is not hard to see that $\bar{f}((0, \infty))=$ $\left(0, \sum a_{i}\right)$. So there exists $x \in(0, \infty)$ such that $\bar{f}(x)=\sum_{i>n} a_{i}$ for $n>1$, which implies that $x=\sum_{i>n} b_{i}$. This yields a contradiction with $\sum_{i>n} b_{i}=\infty$ for all $n \in N$. So the proof of Proposition 2 is complete.

The next theorem states the main result of this work. 
Theorem 2. Let $\left\{a_{n}\right\}$ be a sequence of real numbers such that $0<a_{n+1} \leq$ $a_{n} \leq \sum_{i>n} a_{i}$ for all $n$, and let $\lim _{n \rightarrow \infty} a_{n}=0$. Let $\left\{b_{n}\right\}$ be a sequence of nonnegative real numbers such that $\left\{b_{n}\right\} \ll\left\{a_{n}\right\}$. Then there exists $\alpha \in R$ such that $b_{n}=\alpha a_{n}$ for all $n \in N$.

Proof. Consider the function $f$ defined on $\left[0, \sum_{i=1}^{\infty} a_{i}\right]$ by $f\left(\sum_{I} a_{i}\right)=\sum_{I} b_{i}$. If $\sum a_{n}$ is divergent then $f$ is clearly increasing (by Proposition 1). And if $\sum a_{n}$ is convergent then $\sum b_{n}$ is convergent and again $f$ is increasing (see [6]). Therefore, $f$ is almost everywhere differentiable (see [7, p. 96]). Let $f$ be differentiable at $x \in(0, \infty)$. Set $x=\sum_{I} a_{i}$ with $I \subset N$. Define

$$
h_{n}= \begin{cases}a_{n} & \text { if } n \in N-I, \\ -a_{n} & \text { if } n \in I .\end{cases}
$$

Then

$$
\frac{f\left(x+h_{n}\right)-f(x)}{h_{n}}=\frac{b_{n}}{a_{n}}
$$

for all $n \in N$. Since $f$ is differentiable at $x$, we deduce that $\lim _{n \rightarrow \infty}\left(b_{n} / a_{n}\right)=$ $\alpha$ exists. Assume that there exists $n_{0}$ such that $b_{n_{0}} / a_{n_{0}} \neq \alpha$. Put

$$
A=\left\{n \in N ; \frac{b_{n}}{a_{n}} \geq \frac{b_{n_{0}}}{a_{n_{0}}}\right\} \quad \text { if } \frac{b_{n_{0}}}{a_{n_{0}}}>\alpha .
$$

Then $A$ is a finite set. Therefore, using Proposition 1, there exists an infinite subset $I$ of $N$ such that $\sum_{i \in A} a_{i}=\sum_{i \in I} a_{i}$. So

$$
\sum_{i \in A-I} a_{i}=\sum_{i \in I-A} a_{i}
$$

and since $\left\{b_{n}\right\} \ll\left\{a_{n}\right\}$, we have

$$
\sum_{i \in A-I} b_{i}=\sum_{i \in I-A} b_{i}
$$

This yields, by the definition of $A$,

$$
\frac{b_{n_{0}}}{a_{n_{0}}} \sum_{i \in A-I} a_{i} \leq \sum_{i \in A-I} b_{i}=\sum_{i \in I-A} b_{i}<\frac{b_{n_{0}}}{a_{n_{0}}} \sum_{i \in I-A} a_{i}
$$

Therefore, $\sum_{A-I} a_{i}=\sum_{I-A} a_{i}=0$, which implies that $A=I$, contradicting the fact that $A$ is finite and $I$ is infinite. So $b_{n}=\alpha a_{n}$ for all $n$.

We complete the proof by noticing that if $b_{n_{0}} / a_{n_{0}}<\alpha$ then one can set

$$
A=\left\{n \in N ; \frac{b_{n}}{a_{n}} \leq \frac{b_{n_{0}}}{a_{n_{0}}}\right\} .
$$

Theorem 2 can be interpreted as a result on purely atomic measures. For the nonatomic case, one can consult $[1,8]$. In the next theorem an extension to arbitrary $\sigma$-finite measures is discussed. Notice that the finite case is proved in [6].

Theorem 3. Let $\mu$ be a $\sigma$-finite measure. Assume that the range of $\mu$ is an interval. If $\mu$ is a purely atomic measure, we will assume that the $\mu$-measure of the atoms decreases to 0 . Then any measure $\mu^{\prime}$ such that

$$
\mu(A)=\mu(B) \quad \text { implies } \mu^{\prime}(A)=\mu^{\prime}(B)
$$

is proportional to $\mu$, i.e., there exists $\alpha \in R$ such that $\mu^{\prime}=\alpha \mu$. 


\section{REFERENCES}

1. E. Bolker, Functions resembling quotients of measures, Trans. Amer. Math. Soc. 124 (1966), 292-312.

2. R. Chuaqui and J. Malitz, Preorderings compatible with probability measures, Trans. Amer. Math. Soc. 279 (1983), 811-824.

3. J. A. Guthrie and J. E. Nymann, The topological structure of the set of subsums of an infinite series, Colloq. Math., vol. 55, PNW, Warsaw, 1988, pp. 129-133.

4. S. Leth, A uniqueness condition for sequences, Proc. Amer. Math. Soc. 93 (1985), 287-290.

5. J. Malitz, A strengthening of Leth's uniqueness condition for sequences, Proc. Amer. Math. Soc. 98 (1986), 641-642.

6. J. E. Nymann, A uniqueness condition for finite measures, Proc. Amer. Math. Soc. 108 (1990), 913-919.

7. H. L. Royden, Real analysis, 2nd ed., Macmillan, New York, 1968.

8. C. Villegas, On qualitative probability o-algebras, Ann. Math. Statist. 35 (1964), 1787-1796.

Department of Mathematical Sciences, The University of Texas at El Paso, El Paso, TEXAS 79968-0514

E-mail address: GH00@UTEP 